\title{
Velocity Measurement of Molten Metal Flow at Very High Temperatures
}

\author{
M. Iguchi ${ }^{1}$, H. Kawabata ${ }^{2}$ and Z. Morita ${ }^{3}$ \\ 'Division of Materials Science and Engineering, Graduate School of Engineering, Hokkaido University, \\ North 13, West 8, Kita-ku, Sapporo, Hokkaido, 060-8628 Japan \\ ${ }^{2}$ Division of Materials Science and Processing, Graduate School of Engineering, Osaka University \\ 2-1 Yamadaoka, Suita, Osaka 565-0781 Japan \\ ${ }^{3}$ Professor Emeritus, Osaka University, now with Sumitomo Metal Industries Ltd,, \\ 1-8 Fuso, Amagasaki, Hyogo, 660-0891 Japan
}

(Received October 27, 1998)

\begin{abstract}
Velocimeters currently used to measure the velocity of fluid flows in materials processing are introduced. Particular attention is paid to possible measuring techniques for molten metal flow velocities at very high temperatures.
\end{abstract}

\section{INTRODUCTION}

Fluid flow phenomena in the reactors of current materials processes such as steelmaking at very high temperatures play an essential role in the efficiency of those processes. Various types of velocimeters have been developed for fluids near room temperature /1-6/. Among them pitot tubes, hot wire/film anemometers, laser Doppler velocimeters, propeller type velocimeters, particle imaging velocimetry (PIV) and magnet probes are popular and widely used for model experiments in materials processing. In particular, PIV has become a powerful tool for measuring instantaneous velocity flow fields owing to recent rapid developments in computer science.

Unfortunately, most of the aforementioned velocimeters are not applicable to molten metals at a temperature higher than $1000^{\circ} \mathrm{C}$. It is practically impossible to measure the turbulence characteristics in molten metal flows even at a temperature of around $200^{\circ} \mathrm{C}$. Although some kinds of velocimeters have been developed for molten metal flows, their accuracy is not high, and accordingly, previous numerical results of molten metal flow velocities in actual processes such as ladles did not receive any experimental confirmation. In this article the velocimeters commonly utilized in materials engineering research will be briefly reviewed.

\section{VELOCIMETERS FOR FLUIDS AT AROUND ROOM TEMPERATURE}

\subsection{Pitot tube}

The pitot tube does not measure the velocity of fluid, $v$, directly but detects the dynamic pressure, $p_{d}$, defined as the difference between the total pressure $p_{t}$ and the static pressure $p_{s}$. The dynamic pressure $p_{d}$ is written by

$$
p_{t}=p_{1}-p_{s}=\rho v^{2} / 2
$$

where $\rho$ is the density of fluid.

This method is one of the most accurate methods of measuring the velocity of steady flows. However, the pitot tube is not adequate for measuring the velocity of recirculating flows typical of flows in ladles and converters, because the pitot tube itself disturbs the flow pattern. 


\subsection{Hot wire/film anemometers}

There is a unique relationship between the heat transfer from a heated wire (or a heated film) and the velocity of flow approaching it. Hot wire/film anemometers rely on this relationship and work satisfactorily only when the heat transfer rate is controlled by forced convection. Like pitot tubes, these anemometers are also intrusive.

Hot wire/film anemometers can be used only for fluid flows with a temporally as well as spatially uniform temperature distribution. In addition, the temperature of fluid should be lower than approximately $100^{\circ} \mathrm{C}$. In materials engineering research, hot film anemometers are used to measure the velocity of mercury.

\subsection{Laser Doppler velocimeter (LDV)}

This velocimeter uses the Doppler effect of laser beams reflected from a moving body. Recently many types of laser Doppler velocimeters have been developed and find wide applications. in a variety of engineering fields. This optical technique makes it possible to measure the local instantaneous velocity of tracer particles suspended in the flow and is a kind of nonintrusive method. This method is based on the assumption that the particles, called seeding particles, move with the fluid motion without relative velocity. Such seeding particles must be very small so that they do not disturb the flow pattern and follow the fluid motion completely $/ 4,5 /$.

In materials engineering, an LDV is extensively used in water model experiments for converters, ladles and continuous casing molds. The other velocimeters listed above are not adequate to obtain information on the turbulence characteristics in such flow fields with very high turbulence intensity.

\subsection{Magnet probe (Vives probe)}

A magnet probe, specifically called the Vives probe, works on the basis of Faraday's law 77,8 . According to Weissenfluh $/ 8 /$, if an electric conductor moves through a magnetic field, the conductor has an electromotive force induced in it which is in a direction normal to the magnetic field and the direction of motion. The electro- motive force generates an electric field which is nearly proportional to the intensity of the magnetic field and to the velocity of the conductor. Therefore, it is possible to measure the velocity of the conductor by detecting the electromotive force.

The magnet probe is essentially applicable to molten metal flows at a temperature lower than the Curie point of the permanent magnet $\left(\leq 700^{\circ} \mathrm{C}\right)$. Previous magnet probe measurements for a Wood's metal bath were carried out by Xie et al. /9/ and Iguchi et al. /10/ at a bath temperature lower than $150^{\circ} \mathrm{C}$.

An outline of the magnet probe utilized by Iguchi et al. $/ 10 /$ is illustrated in Figs. 1 and 2 . The original output voltage of the magnet probe was basically very low (approximately $50 \mu \mathrm{V}$ ) under normal conditions, and hence, two amplifiers were necessary. The original voltage was amplified by $10000(100 \times 100)$ times and then recorded on a data recorder. Signals originating from bubbles were removed using a differentiating circuit and a personal computer in a manner as shown in Fig. 3, where $t$ is time and $E v$ and $\mathrm{Er}$ denote the output voltage

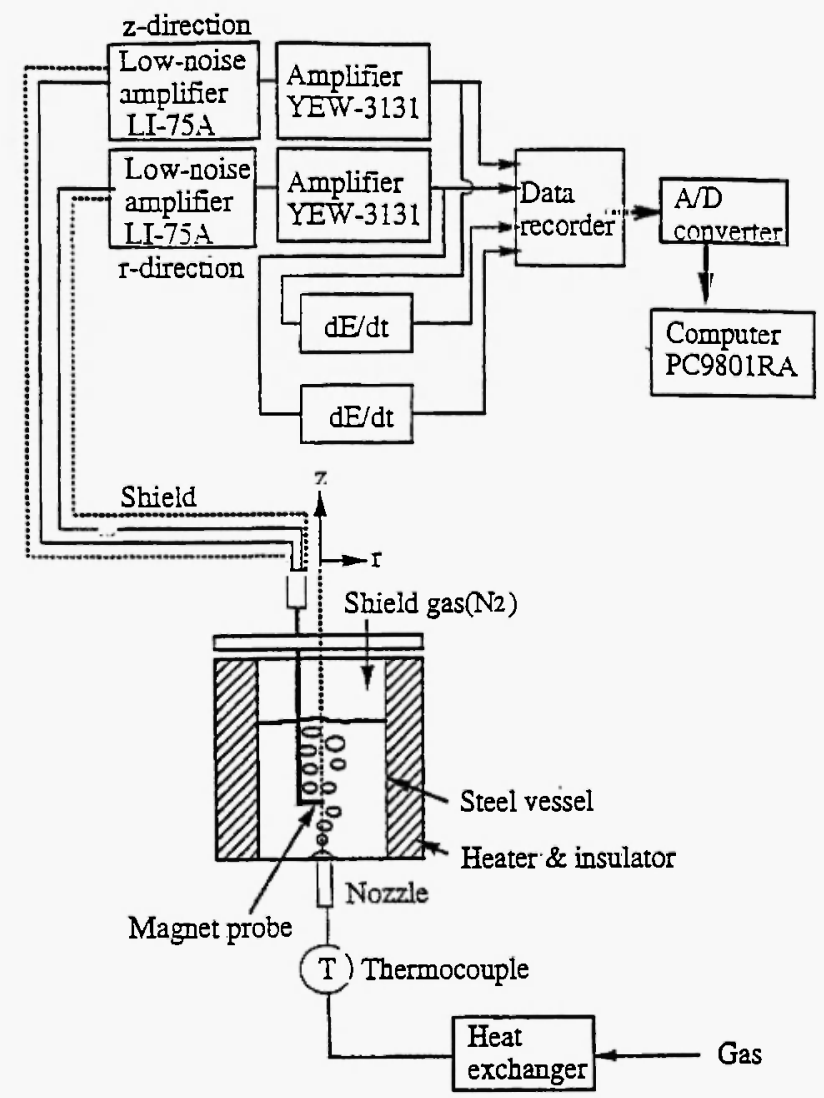

Fig. 1: Experimental apparatus. 


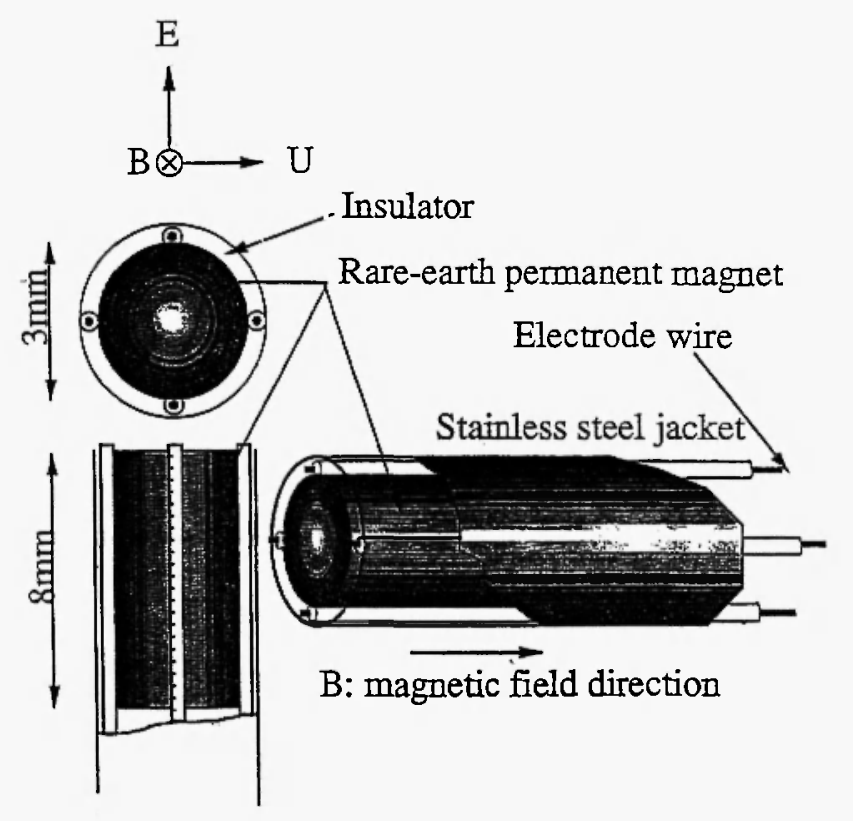

Fig. 2: Details of magnet probe.
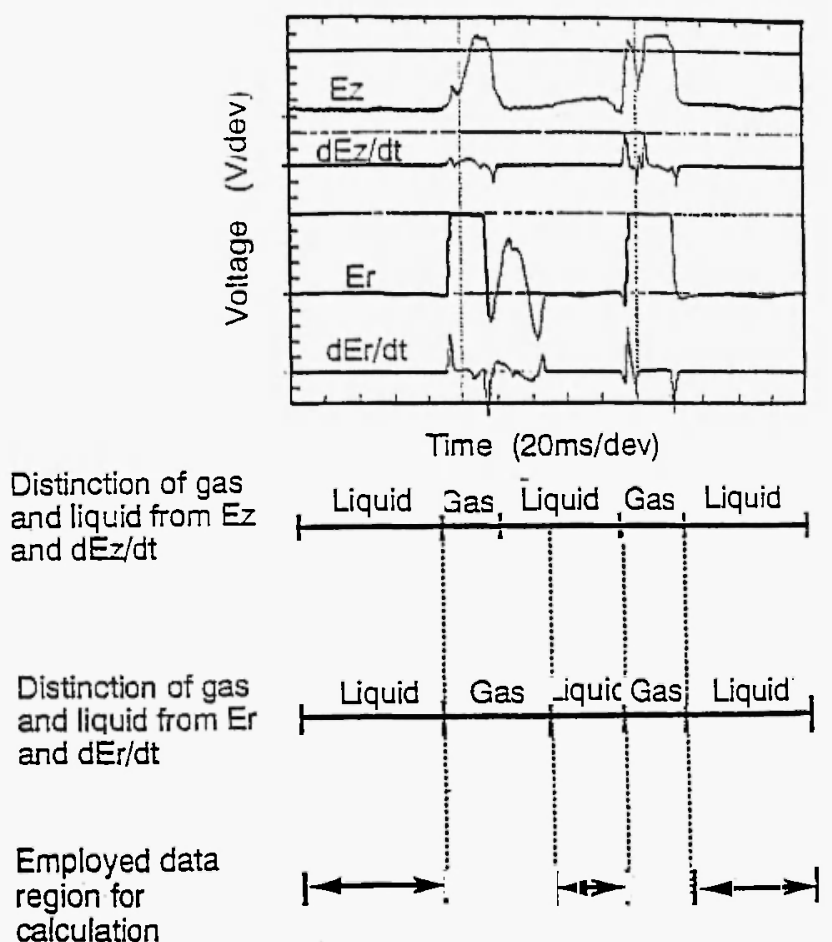

Fig. 3: Discrimination of gas and liquid velocity signals.

values for the axial and radial velocity components, respectively.

\subsection{Propeller type velocimeter}

This velocimeter was originally developed for measuring water flow velocity in models for rivers and canals in civil engineering. The measurement principle of this velocimeter relies on the unique relationship between the rotation speed of a propeller and the velocity of flow approaching the propeller. The smallest diameter is an order of $1 \mathrm{~mm}$, and hence, the spatial resolution is relatively good and suitable for velocity measurement in cold models for continuous casting molds $/ 11$.

\subsection{Particle imaging velocimetry (PIV)}

\subsubsection{Principle}

The technique of particle imaging velocimetry (PIV) has advanced quickly because of the following merits /12-14/:

1) instantaneous whole flow field measurement,

2) contact free (non-intrusive) measurement,

3) easy extraction and processing of physical information through velocity information,

4) PIV is applicabie from slow flows to supersonic flows.

The term 'velocity' used in PIV does not mean exactly the velocity of fluid flow but the velocity of particle motion or the moving velocity of local small fluid elements with small particle concentration distributions. The particle velocity can be taken as the fluid flow velocity only when the particle or particle concentration distribution pattern moves with fluid flow without relative velocity. In applying PIV, good traceability of particle motion to fluid flow should be satisfied.

Whenever any technique of PIV is applied, fluid flow is visualized by one of the existing visualization techniques, and the visualized images are processed after $\mathrm{A} / \mathrm{D}$ conversion.

\subsubsection{Classification of PIV}

The techniques of PIV are classified and briefly explained as follows:

\section{(1) Multiple exposure technique}

(1-1) Path line method

The moving velocity of a particle can be measured 
by dividing the particle path line length with the exposure time.

\section{(1-2) Stroboscopic method}

When particle motions are photographed using a pulse type lighting by a stroboscope, dotted lines of particle images are recorded on a picture. The moving velocity of a particle is measured from the distance between the dotted images and the lighting pulse interval.

(2) Methods using consecutive time step pictures

(2-1)Four consecutive time step particle tracking velocimetry (PTV)

Using consecutive four time step pictures of flows visualized by particle tracers, which are photographed at short time intervals, each particle is tracked and identified, and then the velocities of particles are calculated from the particle movement distances and the time interval. We can identify each particle by judging whether each trajectory of particle motion in the consecutive pictures is smooth or not.

\section{(2-2) Brightness (or concentration) distribution pattern cross-correlation method}

By tracking small local parts with the highest similarity of spatial brightness (concentration) distribution patterns in the two consecutive pictures visualized by small particles like smoke or dyes, the velocity of fluid flow is calculated from the movement distances of the local parts and the time interval using the cross-correlation method.

\section{(3) Binary image cross-correlation method}

We assume that the time interval between the twoconsecutive pictures is short enough and fixed, and that flow velocities in the flow field do not change suddenly in time and space. In other words, the similarity of flow patterns is assumed to be preserved.

Suppose that particle images in a 2-D flow are captured at time $t$ and $t+\Delta t$, as shown in Fig. 4 and that the coordinates of every particle center are calculated already. The principle of particle identification in the two pictures is based on finding out the best similarity in the particle distributions around a reference particle in the first picture and around a candidate particle in the second picture. In order to estimate the similarity in the
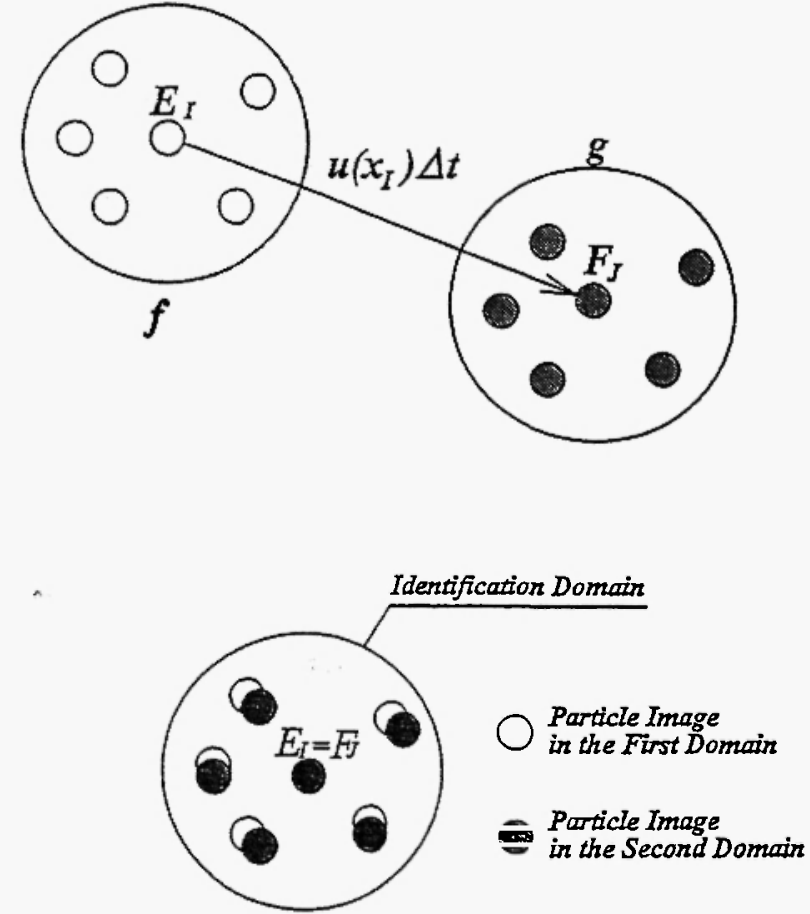

Fig. 4: Movement of particles and overlapping condition.

two particle distribution patterns $\mathrm{F}$ and $\mathrm{G}$, the following cross-correlation coefficient $\mathrm{C}_{\mathrm{fg}}$ can be used /15/.

$$
C_{s_{s}}=\cdot \mathrm{L} /[\mathrm{nm}]^{1 / 2}
$$

where $\mathbf{n}$ and $\mathrm{m}$ are the particle numbers in the correlation domains at time $t$ and $t+\Delta t$, respectively, and $L$ is the summation of logical products in the two subregions $f$ and $g$.

\subsubsection{Application of binary image cross-correlation method}

The binary image cross-correlation method was applied to the flow field in a bottom blown bath /15/. A schematic of the experimental apparatus is shown in Fig. 5 and the velocity vectors in the upper, middle, and lower part of the bath are shown in Fig. 6. These velocity vectors were almost agreed with those measured with an LDV although the results are not shown here. 


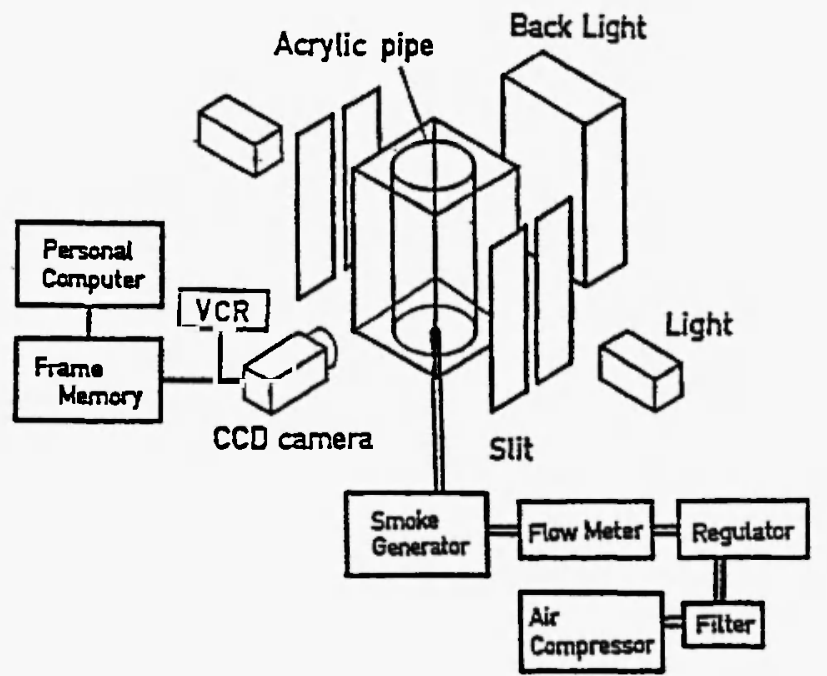

Fig. 5: Schematic display of experimental apparatus.
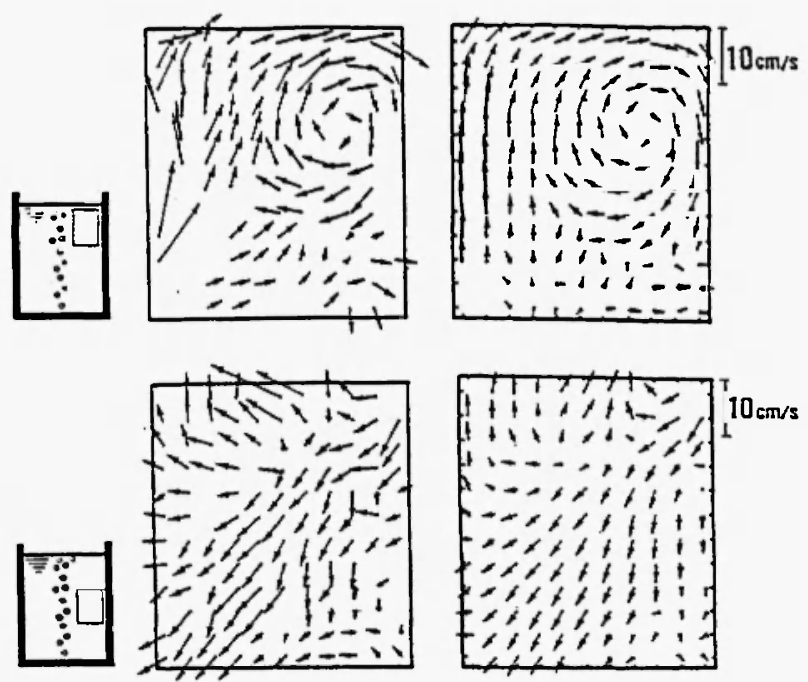

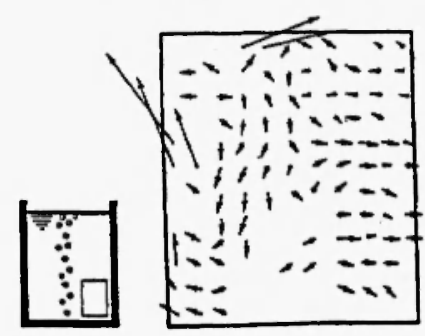

(a)

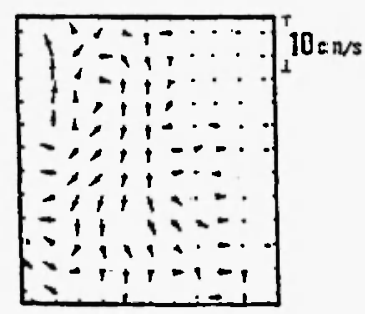

(b)
Fig. 6: Two kinds of velocity distributions measured by image analysis. Gas flow rate $\mathrm{Q}_{\mathrm{g}}=2 \mathrm{~cm}^{3} / \mathrm{s}$, bath depth $\mathrm{H}_{\mathrm{L}}=270 \mathrm{~mm}$; (a) Instantaneous velocity distribution; (b) Time averaged velocity distribution.

\section{VELOCIMETERS FOR MOLTEN METALS AT VERY HIGH TEMPERATURES}

\subsection{Reaction probe}

Velocimeters of which the measurement principle is based on the detection of hydrodynamic drag acting on a solid body are called the reaction probe. Hsiao et al. developed a velocimeter capable of detecting the hydrodynamic drag of a circular disk and measured the liquid velocity in a bath agitated by bottom Ar injection /16/. The reliability of this velocimeter, however, is questionable when it is used in highly turbulent flows, as the drag coefficient $C_{D}$ of the disk changes drastically with a slight change in the attack angle or the incident angle between the disk and the flow direction. Such a shortcoming can be removed by using a sphere instead of a disk, because the drag coefficient of the sphere does not depend on the flow direction.

The mean drag $F_{D}$ for a sphere placed in a stream with the mean velocity $v$ is given by $/ 17-20 /$

$$
F_{D}=C_{12} \cdot\left(\pi D^{2} / 4\right) \cdot\left(\rho \mathrm{v}^{2} / 2\right)
$$

where $D$ is the diameter of the sphere and $\rho$ is the density of fluid. If $C_{D}$ is known, the mean velocity $v$ can be determined from the measured value of the mean drag $F_{D}$.

The principle of velocity measurement with this velocimeter is very simple but many practical problems must be solved before applying it to highly turbulent flows typical of materials processes. The most significant one is the evaluation of $C_{D}$. Information on $C_{D}$ of a sphere placed in a flow field with high turbulence intensity such as a bath agitated by gas injection is not available at present.

The drag coefficient $C_{D}$ is generally represented as a function of three variables $/ 21,22 /$ :

$$
\begin{aligned}
& C_{D}=f(\operatorname{Re}, \quad T u, \lambda / D) \\
& \operatorname{Re}=v D / v \\
& T u=v^{\prime}{ }_{r m s} / v \\
& v^{\prime}{ }_{r m s}=\left(v^{\prime 2}\right)^{1 / 2}
\end{aligned}
$$


where $\operatorname{Re}$ is the Reynolds number, Tu is the turbulence intensity, $\lambda$ is the turbulence scale, $v$ is the mean velocity, $v$ is the kinematic viscosity of fluid, $v^{\prime}{ }_{r m s}$ is the root-mean-square (rms) value of the turbulence component $\mathrm{v}^{\prime}$.

The drag coefficient $C_{D}$ becomes small with an increase in Tu. Figure 7 shows $C_{D}$ values determined from pressure measurements for various $\mathrm{Tu}$ values against the Reynolds number $\operatorname{Re} / 23 /$. The solid line denotes the standard drag coefficient $\mathrm{C}_{\mathrm{Ds}}$ recommended by Clift et al. $/ 19 /$.

Figure 8 shows $C_{D}$ data for $T u=5 \%$. The measured values of $C_{D}$ for different turbulence scales agreed well with one another, which fact revealed that $C_{D}$ was insensitive to the relative turbulence scale $\lambda / \mathrm{D}$. The value of the Reynolds number at which $C_{D}$ departed from the

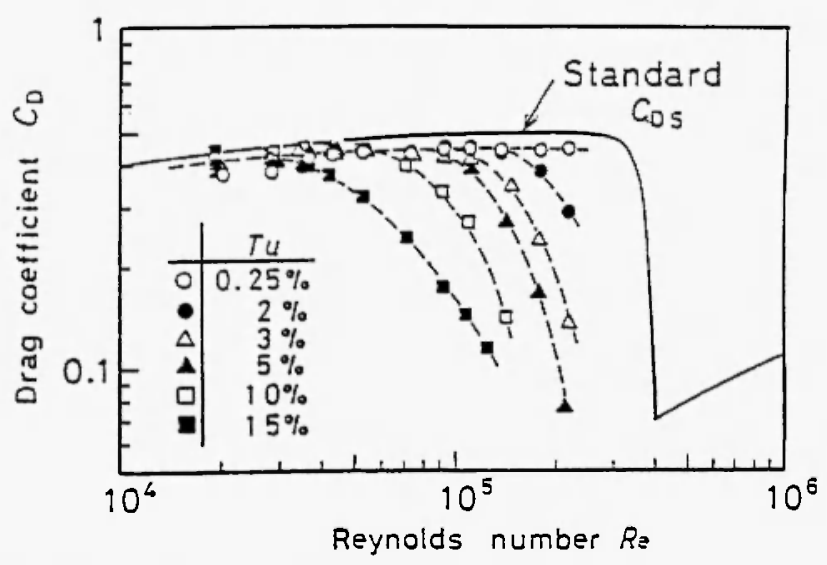

Fig. 7: Drag coefficient of sphere as a function of $\mathrm{Re}$ and Tu.

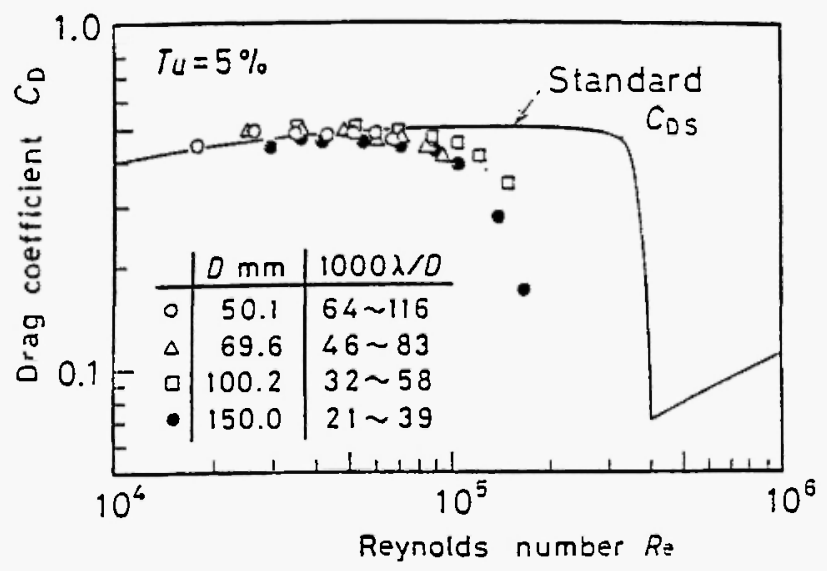

Fig. 8: Drag coefficient of sphere as a function of $\mathrm{Re}$ and $\lambda / D$.
$C_{D s}$ curve for a given Tu was defined as the critical Reynolds number $\mathrm{Re}_{\mathrm{d}}$. Figure 9 shows the relationship between $\mathrm{Re}_{\mathrm{d}}$ and $\mathrm{Tu}$. In the hatched region $\mathrm{C}_{\mathrm{D}}$ followed $C_{D s}$, while in the remaining region $C_{D}$ changed in a complex manner depending on $\mathrm{Tu}$ and $\mathrm{Re}$ as shown in Fig. 7.

Figure 10 shows a schematic diagram of the sphere velocimeter $123 \%$. This one-dimensional velocimeter can detect the velocity only when the flow direction is specified.

Concerning the choice of the sphere diameter D, the following three conditions should be taken into consideration $/ 23 /$.

1) The diameter $D$ is desired to be larger than the

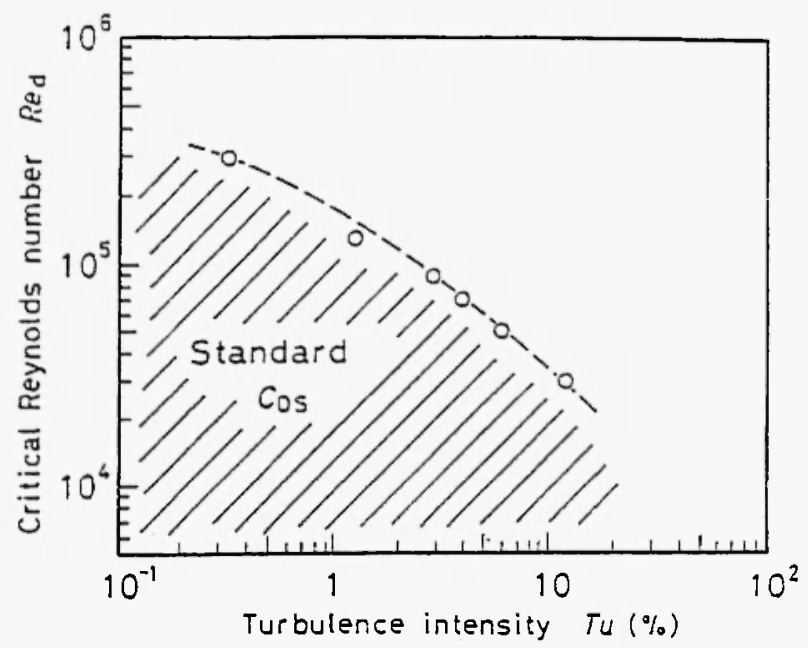

Fig. 9: Relation between $\mathrm{Re}_{\mathrm{d}}$ and $\mathrm{Tu}$.

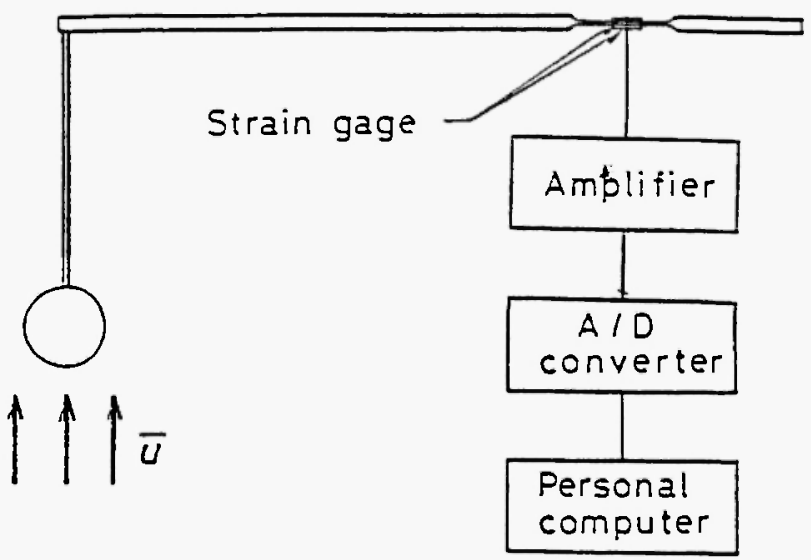

Fig. 10: Velocity probe and the data acquisition system. 
turbulence scale $\lambda$ as much as possible.

2) In order to measure the local mean velocity, a smaller diameter $\mathrm{D}$ should be chosen as long as the drag is precisely detected.

3) It is desirable to choose $D$ in such a way that the drag coefficient $C_{D}$ obeys the standard drag coefficient $C_{D s}$. That is, even if Tu is large, Re should fall in the hatched region in Fig. 9.

The reaction probe is useful for measuring the velocity of molten metal flows near the bath surface in the absence of wave motions. Separation of the drag forces acting on the sphere and its supporting rod becomes difficult as the immersion depth increases.

\subsection{Karman vortex probe}

When a long circular cylinder is immersed in a free stream with its axis perpendicular to the flow direction as shown in Fig. 11, the flow around the cylinder is governed by the Reynolds number defined in Eq. (5) $/ 17,22 /$.

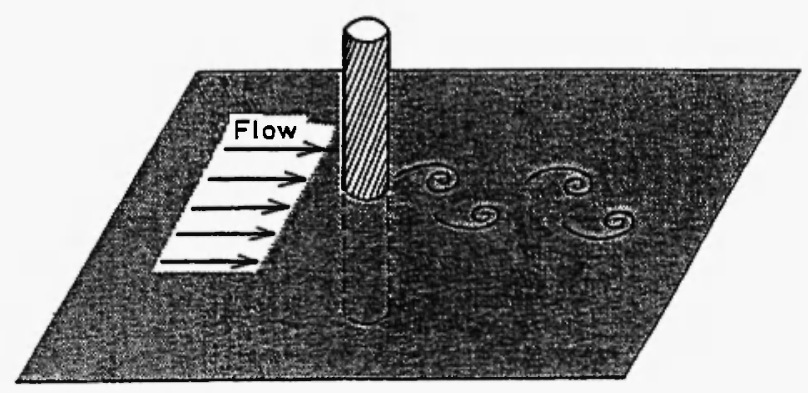

Fig. 11: Schematic of Karman's vortex street.

If the Reynolds number, $\mathrm{Re}$, is larger than approximately 40 , vortices are shed in turn from the cylinder. These very regular patterns are called Karman's vortex streets. In relation to the shedding of Karman's vortices, a periodical force with a direction perpendicular to the flow direction is exerted on the cylinder, and hence, the cylinder begins to oscillate in that direction. The oscillation frequency of the cylinder is the same as the shedding frequency of Karman's vortex streets.

The shedding frequency of Karman's vortex streets, $f_{\text {, }}$ is correlated in terms of the Strouhal number, St, described by

$$
S t=f D / v
$$

It is widely known that measured St values are almost constant over a wide Reynolds number range of approximately $3 \times 10^{2}$ to $2 \times 10^{5} / 17,24 /$. When the Reynolds number falls in this range, the velocity of fluid approaching the circular cylinder, $\mathrm{v}$, can be readily determined by using this relation and by detecting the shedding frequency.

The Karman vortex probe shown in Fig. 12 consists of three parts 125/: the cylindrical test cylinder for generating Karman's vortex streets, the supporting rod which is designed to hold the test cylinder, and the shedding frequency detection unit. At the present stage, sialon is the most suitable for the test cylinder used in molten steel flow of a temperature of approximately $1600^{\circ} \mathrm{C}$. This material has a density of $3.25 \mathrm{~g} / \mathrm{cm}^{3}$ and high resistivity against thermal shock.

This probe was calibrated in the following manner. Wood's metal of a melting temperature of $47^{\circ} \mathrm{C}$ was melted in a cylindrical vessel with an inner diameter of $360 \mathrm{~mm}$ and a height of $220 \mathrm{~mm}$. The bath depth was

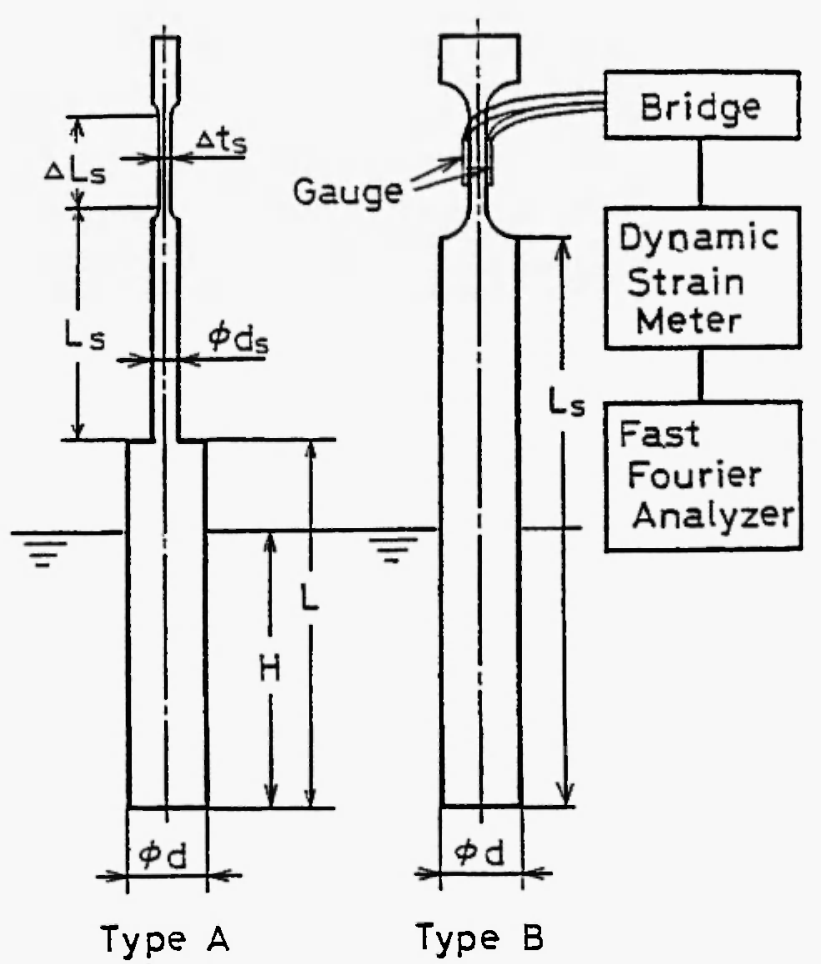

Fig. 12: Schematic of Karman vortex probe and signal processing unit. 
$150 \mathrm{~mm}$ and the temperature was $80^{\circ} \mathrm{C}$. On the other hand, steel was melted in a cylindrical crucible with an inner diameter of $170 \mathrm{~mm}$ and a height of $300 \mathrm{~mm}$ at a temperature of $1550^{\circ} \mathrm{C}$ using an induction furnace. The bath depth was $220 \mathrm{~mm}$. The rotation arm was driven by a variable-speed motor and the rotation radius of the probe was set to $120 \mathrm{~mm}$ for the molten Wood's metal bath and $45 \mathrm{~mm}$ for the molten steel bath. The submerged depth of the test cylinder was $50 \mathrm{~mm}$ for each bath.

The output signal of the dynamic strain meter and its power spectrum obtained by the FFT analyzer for molten steel flow are shown in Fig. 13. The frequency indicated by the symbol $\nabla$ is regarded as the shedding frequency.

Figure 14 compares the experimental results for the molten Wood's metal and molten steel. The measured values for the two systems were in good agreement, and hence, the $\mathrm{k}$ value of 0.15 originally obtained for the molten Wood's metal was also applicable to the molten steel. The erosion and deformation of the test cylinder were negligible although the cylinder was submerged in the molten steel without preheating.

Velocity measurements were also carried out by changing the rotation speed of the rotation arm stepwise. The results were satisfactory, as shown in Fig. 15.

Figure 16 shows the velocity of molten steel flow measured at the meniscus of an actual continuous casting mold. At a casting speed of $3 \mathrm{~m} / \mathrm{min}$ molten steel flow velocity is compared well with that of water flow in a water model of the same dimensions. This fact partly supports the adequacy of the Karman vortex probe.

\subsection{Magnet probe for continuous casting mold}

Julius et al. $/ 26 /$ used multiple magnet sensors mounted into the wall of a continuous casting mold to measure the molten steel flow velocity near the wall. Each sensor consists of a permanent magnet and two highly sensitive detectors without direct steel contact. The measurement principle is the same as described for the Vives probe.

The electromagnetic field of the induced eddy cur-
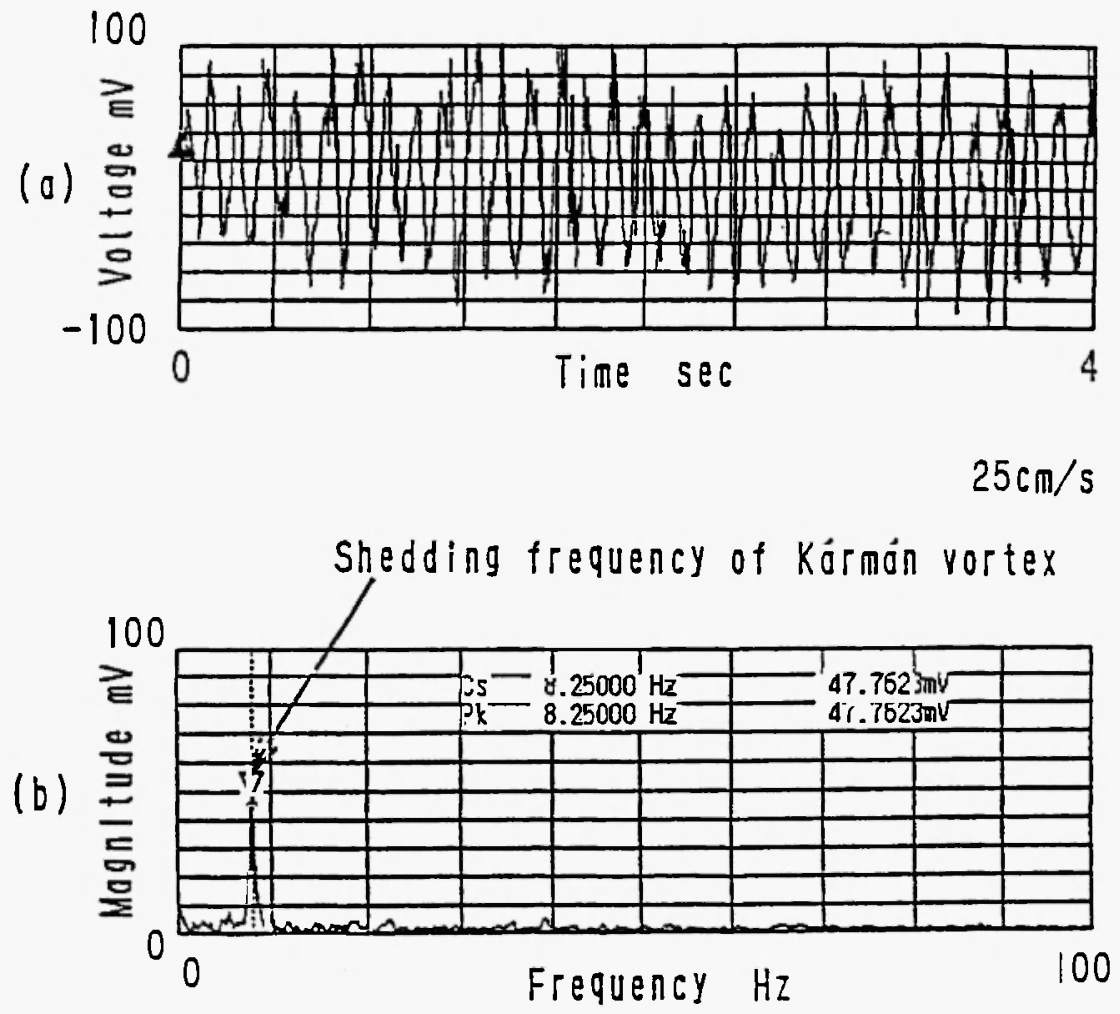

Fig. 13: Output signal of strain meter and its power spectrum for Wood's metal bath. 


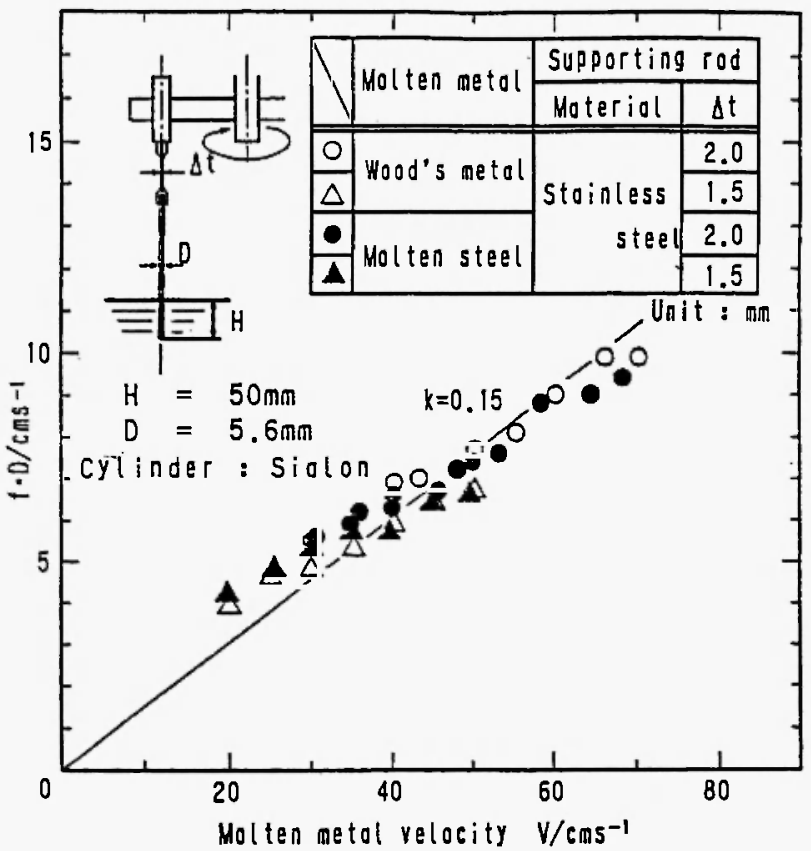

Fig. 14: Relation between molten metal velocity and the product of shedding frequency and cylinder diameter. (Comparison between Wood's metal data and molten steel data)

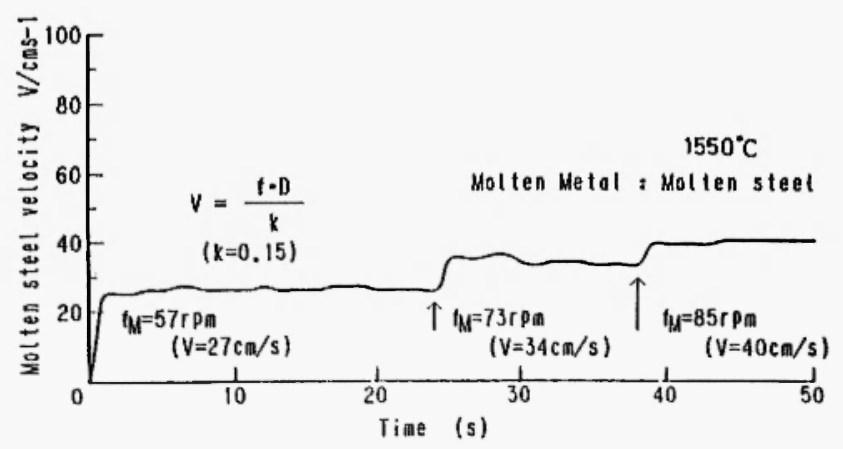

Fig. 15: Molten steel velocity with time.

rents can be measured using appropriate detectors. The strength of the measured field is proportional to the flow velocity but the calibration of the sensors is very difficult. Julius $e t$ al. determined the time delay $\tau$ between the output signals of the two detectors to avoid calibration. The time delay $\tau$ can be obtained by using the cross-correlation method. The mean velocity $v$ is calculated from

$$
\mathrm{V}=\mathrm{L}_{\mathrm{s}} / \tau
$$

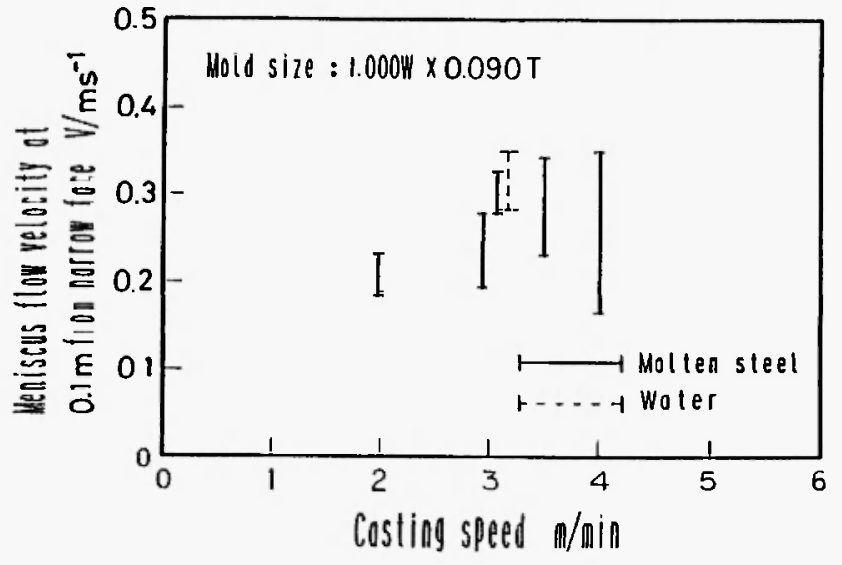

Fig. 16: Molten steel velocity at the meniscus of continuous casting mold.

where $L_{s}$ is the distance between the two sensors.

\subsection{Melting probe}

Mikrovas and Argyropoulos /27/ developed a unique probe by focusing on the melting rate of a metal sphere immersed in molten metal flow with the same chemical composition and applied it to measure the velocity of molten aluminum flow in an $\mathrm{AC}$ induction furnace at a temperature of approximately $750^{\circ} \mathrm{C}$. The results were compared with their own numerical predictions. They found that the experimental results were in good agreement with the predictions. This probe, however, is very sensitive to the fluctuation of molten metal temperature just like hot wire/film anemometers. Accordingly, it is necessary either to predict the temperature fluctuation before the usage of the probe or to measure it together with the velocity. In addition, the effect of the turbulence intensity on the heat transfer from a solid metal sphere immersed in molten metal flow of the same kind must be taken into consideration.

\subsection{Particle imaging velocimetry}

The velocity of flow on the surface of a molten metal bath can be measured by recording tracer motions on the bath surface and subsequently processing the tracer images by using one of the aforementioned PIV methods.

Molten metal flow velocities in the inner part of the 
bath of reactors and continuous casting molds can also be measured provided that the movement of tracers are detected by, for example, an X-ray fluoroscope.

\section{CONCLUDING REMARKS}

Recent rapid developments in numerical simulation techniques for fluid flow phenomena have attracted the interest of researchers and engineers in materials engineering as well. Numerical results are beginning to be used to design actual materials processes. In order to validate the numerical results, however, velocimeters applicable to molten metal flows at very high temperatures are necessary. Further work is expected to develop such velocimeters.

\section{REFERENCES}

1. B. Lewis, R.N. Pease and H.S. Taylor, Physical Measurements in Gas Dynamics and Combustion, Princeton University Press, 1954; 6.

2. B.J. Clark and M. Gardner, Measurement Systems Application and Design, McGraw-Hill, 1975.

3. O.D. Ernest, Measurement Systems, Application and Design, McGraw-Hill, 1975.

4. F. Durst, A. Melling and J.H. Whitelaw, Principles and Practice of Laser-Doppler Anemometry, Academic Press, London, 1976.

5. Association for the Study of Flow Measurements, Fundamentals and Applications of LDV: Nikkan Kogyo Shinbun Co. Ltd., 1980.

6. J. Visualization Soc. Jpn., Handbook of Flow Visualization, Asakura Book Co. Ltd., Tokyo, 1986.

7. T. von Weissenflue, Int. J. Heat Mass. Transfer, 28, 1563 (1985).

8. R. Ricou and C. Vives, Int. J. Heat Mass Transfer, 25, 1579 (1982).

9. Y. Xie, S. Orsten and F. Oeters, ISIJ Int., 32, 66
(1992).

10. M. Iguchi, H. Tokunaga and H. Tatemichi, Metall. Mater. Trans. B, 28, 1053 (1997).

11. K. Tozawa, S. Idogawa, S. Nakata and K. Sorimachi, CAMP-ISIJ, 9, 604 (1996).

12. R.J. Adrian, Annu. Rev. Fluid Mech., 23, 261 (1991).

13. F. Yamamoto, M. Iguchi, J. Ohta and A. Szajner, Proc. Int. Conf. on Fluids Eng., Chosun University, Kwangju, Korea, 1994; 11-39.

14. K. Okamoto, T. Saga, S. Nishio and T. Kobayashi, J. Visualization Soc. Jpn., 17, 239 (1997).

15. T. Uemura, M. Iguchi, W. Mizukoshi and Z. Morita, Proc. $6^{\text {th }}$ Int. Iron and Steel Congress, Nagoya, 1990; 1-477.

16. T.C. Hsiao, T. Lehner and B. Kjellberg, Scand. J. Metall., 9, 105 (1980).

17. T. Ikui and M. Inoue, Dynamics of Viscous Fluids, Rikougaku Co. Ltd., 1987; 76.

18. E. Achenbach, J. Fluid Mech., 34, 625 (1968).

19. R. Clift, J.R. Grace and M.E. Weber, Bubbles, Drops and Particles, Academic Press, 1978; 112.

20. H.L. Dryden, G.B. Schubauer, W.C. Mock, Jr. and H.K. Skramstad, NACA Rep., 581, 109 (1937).

21. Handbook of Fluid Dynamics, Japan Society of Fluid Dynamics, Maruzen Co. Ltd., 1987; 231.

22. H. Schlichting, translated by J. Kestin, BoundaryLayer Theory, $7^{\text {th }}$ ed., McGraw-Hill, 1979; 313.

23. M. Iguchi, H. Kawabata, $Y$. Demoto and $Z$. Morita, ISIJ Int., 34, 461 (1994).

24. L.D. Landau and E.M. Lifsitz, translated by $\mathrm{H}$. Takeuchi, Hydrodynamics, Tokyo Tosho Co. Ltd., 1973; 1-26.

25. M. Iguchi, M. Takeuchi, H. Kawabata, K. Ebina and Z. Morita, Trans. JIM, 35, 716 (1994).

26. E. Julius, H. Haubrich, P. Andrzejewski and D. Gotthelf, Proc. Int. Cong. Electromagnetic Processing of Materials, Paris-Lu-Defense, 1997; 5-67.

27. A.C. Mikrovas and S.A. Argyropoulos, Metall. Trans. B, 24, 1009 (1993). 